\title{
A new algorithm for wireless network nodes effectiveness
}

\author{
XiangZhong Wang Moyi Duan \\ Software College \\ Nanjing railway vocational and technical college \\ Nanjing , China \\ e-mail:duanmoyi@163.com
}

\begin{abstract}
According to the network congestion caused by node failure, presents a measuring node effectiveness evaluation index. The index for the long correlation properties of the actual flow, respectively using the autoregressive moving average (Auto-Regressive and Moving Average, ARAMA) model and linear fractional stable noise (Stable Noise Linear Fractiona, SNLF) model prediction method of traffic arrival, and through simulation experiments to study the relationship between the index and the average arrival rate, between the results show that, when the average arrival rate is lower the ARAMA model performance is better, and better performance of SNLF model.

Keywords: network node; long; autoregressive moving average model; linear fractional stable noise model

\section{Introduction}

Reliability is the characteristic of the network information system can complete the function under specified conditions and within the prescribed period of time. Reliability is an important index to evaluate the communication network planning and performance, is the construction and operation of all the network information system. Generally includes a plurality of network survivability, survivability and effectiveness and reliability of communication network, relates to the network communication equipment, topology structure, communication protocol, etc.. The computer network and communication network are inseparable, they are completely fused.
\end{abstract}

Reliability is mainly manifested in the aspects of hardware reliability, software reliability, human reliability, environmental reliability. The most intuitive and common hardware reliability. Software reliability is defined within the prescribed time, probability of successful operation of the program. Reliability refers to the probability of personnel to successfully complete the job or task. Personnel reliability plays an important role in the whole system reliability, because most cause of system failure was caused by human error. Human behavior is influenced by physiological and psychological, influenced by the technical proficiency, responsibility and moral quality of the. Therefore, staff education, training, training and management and reasonable man-machine interface is an important aspect of improving reliability. Environment reliability refers to the environment, to ensure the successful operation of the probabilistic network. The environment here is mainly refers to the natural environment and electromagnetic environment.

The reliability of computer network is one of the inherent characteristics of computer network system, it is shown that a computer network system according to the user's requirements and design objectives, level correctly perform their functions. Computer network reliability and network software reliability, the hardware reliability and the environment. The reliability of computer network should contain the following 3 elements.

(1) the failure-free operation time

The reliability of computer network is reflected in the running stage, with "no time" failure to measure. Because of the randomness of selecting network environment, network program path and the attack, software failure is a random event, so the failure-free operation time belongs to random variables.

(2) environmental conditions

Environmental conditions of operation environment of computer network. All it needs to involve the network run-time system support elements, such as hardware, software, control system, may be subject to attack, the protection and control procedures. Information on the reliability of the network under different conditions are different.

(3) the function 
The reliability of computer network and the provisions of the relevant task and function. Due to the different tasks, operational profile information network will be different, then call the network module is different, its reliability may be different, so to correct measure of reliability of computer network, we must first define its mission and function.

The reliability of network information system mainly has three kinds: survivability, survivability and effectiveness.

Survivability refers to the reliability of the system in the destruction of the. For example, part of the line or node failure, whether the system can still provide some level of service. The survivability can effectively avoid all kinds of disasters (such as war, earthquake) large area paralysis caused by events.

Survivability is the reliability of the system in random destruction under. Survivability mainly reflects the effect of random failure and the network topology structure of system reliability. Here, random destruction refers to system components for natural aging caused by the natural failure.

Effectiveness is a reliability based on business performance. Effectiveness is mainly reflected in the network information system component failure case, meet the performance requirements of the degree of business. For example, the failure of a network component does not result in connectivity fault, but caused quality index decreased, increased, average delay line congestion phenomenon.

Congestion is a persistent overload of the network, the users of the cyber source (including the link bandwidth, storage space and processing capacity, etc.) the demand exceeds the inherent capacity. On the system structure of Internet, the occurrence of congestion is the inherent attribute. Because there is no licensing negotiation mechanism and request in advance resource sharing network, several IP packet arrives at the router at the same time, the possibility and expect the same output port forwarding is, apparently, not all packets can also accept the deal, there must be a service order, intermediate nodes cache block waiting for service to provide some protection. However, if this situation has a certain continuity, when the cache space is depleted, the router only packet discarding. In this continuous overload condition, the network performance will decline sharply.

The hypothesis network from node a to B multiple paths are up to, as shown in figure 1 . One, 1, 2,.., m said the multiple paths and edge phase a, Max_out_flow (a) said node a maximum output flow, Input_flow (a) said node a actual arrival traffic, Out_flow $(\mathrm{a}, \mathrm{m})$ indicates that the actual output flow adjacent edge on M. Remove the A to the B office that a a phase limb still.

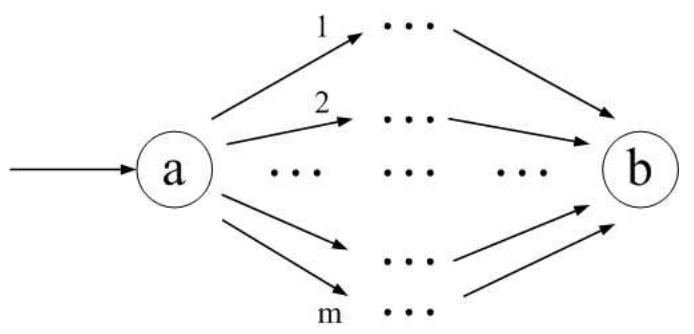

Figure 1 simulation network topology

Definition in a network node a to remove the associated boundary $m$ is effective for:

$\delta(a, m)=\frac{\text { Input_flow }(a)-O u t \_f l o n(a, m)}{\text { Max_out_flow }(a)-O u t \_f l o n(a, m)}$

If $\delta(\mathrm{a}, \mathrm{m})=0$ side $\mathrm{m}$ is not the other side forward; if the $0<\delta(\mathrm{a}, \mathrm{m})<1$ representation of flow only partial side $\mathrm{m}$ is the other side forward; if $\delta(\mathrm{a}, \mathrm{m}) \geq 1 \mathrm{~m}$ flow could all be the other side forwarding. The effectiveness of the node a can be defined as follows:

$$
\delta(a)=E\left(\sum_{m} \delta(a, m)\right)
$$

This indicated that in turn to remove the associated node a side $\mathrm{m}$, and the validity and expectations. 


\section{Calculation of the network node availability}

From the above definition of validity of network nodes can be seen, the maximum output flow and node due to factors of service rate, bandwidth, and the Max_out_flow in the network environment under the condition of fixed (a) values can be regarded as constant, so the solution is the key to the input traffic node Input_flow (a).

In recent years, the study found, the actual traffic exhibits long-range dependence obviously, so here with longrange to study next time reaches the flow Input_flow (a) changes, and establish effective evaluation for network node model. This paper was studied using two methods: the first method using wavelet transform, the improved ARAMA model to study, second methods using SNLF model.

\section{A ARAMA model}

If flow y $(T)$ is composed of an input sequence $x(T)$ excitation of a linear system $H(z)$ output, $H(z)$ is a linear causal invariant discrete time system, the unit sampling response of $H(z)$ is deterministic. The ARAMA model of $[11,12]$ definition, $\mathrm{y}(\mathrm{T})$ and $\mathrm{X}(\mathrm{T})$ has the following relationship between:

$$
y(t)=-\sum_{r=1}^{p} \alpha_{r} y(t-r)+\sum_{r=0}^{q} \beta_{r} x(t-r)
$$

Among them, $\beta 0=1$.

The ARAMA model is the autoregressive (Auto-Regressive, AR) model and moving average (Moving Average, MA) model, type (3) can be as follows the autoregressive model of order P, can be seen as the moving average model obeys the Q order, is mainly used to describe the short flow. But due to the actual flow with long correlation and scaling behavior, if the direct use of ARAMA model to predict, will produce great error, so here we use wavelet transform [13-15] long-range dependence traffic for processing, and then predict the next flow state by using ARAMA model. The effectiveness of the network node of a can be obtained according to the following algorithm:

Step 1 when a certain time t, using the formula (4) shows the wavelet transform to the node a actual flow y (T) decomposition, each layer of the wavelet coefficients of $\mathrm{Dj}, \mathrm{K}$ and approximate coefficients $\mathrm{Aj}, \mathrm{K}$, wherein, $\mathrm{K}$ represents the number of wavelet coefficients in each layer;

$$
\left\{\begin{array}{l}
D_{j, k}=\frac{\sqrt{2}}{2}\left(A_{j+1,2 k}-A_{j+1,2 k+1}\right) \\
A_{j, k}=\frac{\sqrt{2}}{2}\left(A_{j+1,2 k}+A_{j+1,2 k+1}\right)
\end{array}\right.
$$

Step 2 second, using wavelet coefficients Dj, K ARAMA model initialization, parameter estimation of $\alpha$ R (J) $(\mathrm{r}=1,2, \ldots, \mathrm{P})$;

Step 3 using the FIR filter, the output of the approximate MA (q) process, and the estimation of parameter $\beta$ R (J) $(\mathrm{r}=0,1, \ldots, \mathrm{Q})$;

Step $4 \quad j=j+1$, jump to step 2, repeat the estimation of $\alpha R(J)$ and $\beta R(J)$, maximum level until the decomposition;

Step 5 At the same time, according to the step $5 \alpha \mathrm{R}(\mathrm{J})$ and $\beta \mathrm{R}(\mathrm{J})$ parameters of $\mathrm{P}$ and $\mathrm{q}$;

Step 6 by formula (3) calculating the wavelet coefficient D 'new j, k;

Step 7 finally, based on the approximate coefficient Aj, wavelet coefficients of D 'K and calculated J, K, using the inverse wavelet transform predictive y $(\mathrm{T})$ under a state at the time of $\mathrm{Y}(\mathrm{t}+1)$, namely type (1) in the Input_flow (a);

Step 8 by type (1) and (2) computing node availability of $\delta$ a (a).

If node a the actual arrival rate obeys the FBM model from the literature, [16], distribution function of queue length for: 


$$
\begin{aligned}
& F(X<x)= \\
& 1-e^{\frac{(C-m)^{2 H}}{2 a m H^{2 H}(1-H)^{2-2 H}} \cdot x^{(2-2 H)}}
\end{aligned}
$$

The type (5) can be obtained for the derivation of probability density function:

$$
\begin{aligned}
& P(x)= \\
& \frac{(2-2 H)(C-m)^{2 H} x^{1-2 H}}{2 a m H^{2 H}(1-H)^{2-2 H}} e^{-\frac{(C-m)^{2 H}}{2 a m H^{2 H}(1-H)^{2-2 H}} \cdot x^{(2-2 H)}}
\end{aligned}
$$

The actual average length of flow is:

$$
\begin{aligned}
& E(\text { Input_flon }(a)) \\
& =\left(\frac{2 \omega_{H} H^{2 H}(1-H)^{2-2 H}}{(C-v)^{2 H}}\right)^{2-2 H} \Gamma\left(\frac{1}{2-2 H}+1, \frac{b^{2-2 H}(C-v)^{2 H}}{2 \psi^{2 H} H^{2 H}(1-H)^{2-2 H}}\right)
\end{aligned}
$$

Among them, is the largest variance coefficient, $\mathrm{C}$ for the effective bandwidth of the system, $\mathrm{B}$ as the buffer size, $\mathrm{V}$ is the average arrival rate of flow, each Gamma function defined for Matlab; $\mathrm{H}$ for the Hurst parameter, when $\mathrm{H}$ is $[0,0.5]$ traffic exhibits the correlation between short, when $\mathrm{H}(0.5,1], \mathrm{C}$. mean flow with long-range dependence.

\section{B SNLF model}

Suppose that $\mathrm{y}(\mathrm{T})$ for t node a to flow, defined by the literature [17]:

$$
y(t)=v t+\xi x(t)
$$

Among them, $\mathrm{V}$ is the average arrival rate of flow, and for the scale factor, $\mathrm{X}(\mathrm{T})$ with symmetric $\alpha$ - fractal stable motion stability boundary (LFSM), defined as:

$$
L_{\alpha, H}(t)=\int_{-\infty}^{\infty} f(t, x) \cdot M(d x)
$$

$\mathrm{M}(\mathrm{DX})$ is Lebesgue (Lebesgue) alpha stable random measure control measure, alpha is the characteristic index, said the network traffic burst degree. The core function of $F(T, x)$ is defined as:

$$
\begin{aligned}
& f(t, x)= \\
& \phi\left[(t-x)_{+}{ }^{H-1 / \alpha}-(-x)_{+}{ }^{H-1 / \alpha}\right]+ \\
& \varphi\left[(t-x)_{-}{ }^{H-1 / \alpha}-(-x)_{-}{ }^{H-1 / \alpha}\right]
\end{aligned}
$$

Where phi and phi, parameters for any real constant.

Consider the node a with constant service rate $\mathrm{W}$, queue occupancy for:

$q(t)=y(t)-w t \geq 0$

If the discarded beyond the arrival probability envelope process flow, then $q(T)$ of the envelope:

$\hat{q}(t)=\hat{y}(t)-w t \geq 0$

Then:

$$
\hat{q}(t)=K|\alpha| \sigma_{1} t^{H}-(w-v) t, t \geq 0
$$

By literature [18]: 


$$
\begin{aligned}
\sigma_{1}^{\alpha} & =\left(|\theta|^{\alpha}+|\psi|^{\alpha}\right) \int_{0}^{\infty}\left((1+x)^{H-1 / \alpha}-x^{H-1 / \alpha}\right)^{\alpha} d x \\
& +\int_{0}^{1}\left|\theta(1-x)^{H-1 / \alpha}-\psi x^{H-1 / \alpha}\right|^{\alpha} d x
\end{aligned}
$$

Among them, theta and psi for any real constant.

The average length of the operations available for actual arrival flow:

Input_flow $(a)$

$=(1-H)\left[\frac{H^{H} K|\alpha| \sigma_{1}}{(w-v)^{H}}\right]^{\frac{1}{1-H}}$

Among them, K y (T) more than (T) probability.

\section{Mathematical simulation}

In order to verify the effectiveness of the proposed methods, here set up network topology shown in Figure 1 in the NS2, long flow and the program generates the simulation verification. The simulation data based on the fractal Brown motion (Fractional Brownian Motion, FBM) model of [16], average arrival rate was $2279 \mathrm{~kb} / \mathrm{sec}$, the number of edges $m=10$ node a association. At the same time, the simulation results in MATLAB analysis, evaluation of the effectiveness of the network node.

The first program produced 2000 long data (the degree of correlation with Hurst parameters to represent, abbreviated as $\mathrm{H}$, when $\mathrm{H}$ is $[0,0.5]$ flow correlation between short, when $\mathrm{H}(0.5,1]$, mean flow with long-range dependence; here $\mathrm{H}=0.9$ ), and the former 1000 data as a priori information. The recursive formula for decomposition of these 1000 data using wavelet transform, the wavelet coefficients of $\mathrm{Dj}, \mathrm{K}$ and approximate coefficients $\mathrm{Aj}, \mathrm{K}$, where $\mathrm{k}$ represents the number of wavelet coefficients in each layer.

The results are as shown in figure 2. As you can see from Figure 2, the above two kinds of prediction results of long data and program produces relatively close, calculate the standard deviation of ARAMA 6.89, SNLF 6.23. The two method has certain prediction accuracy.

At the same time, are simulated in MATLAB, evaluation of the effectiveness of the network node. The type (7) and (15) respectively by type (1) and (2), $\delta$ node effectiveness can be obtained by the two methods (a). Assume

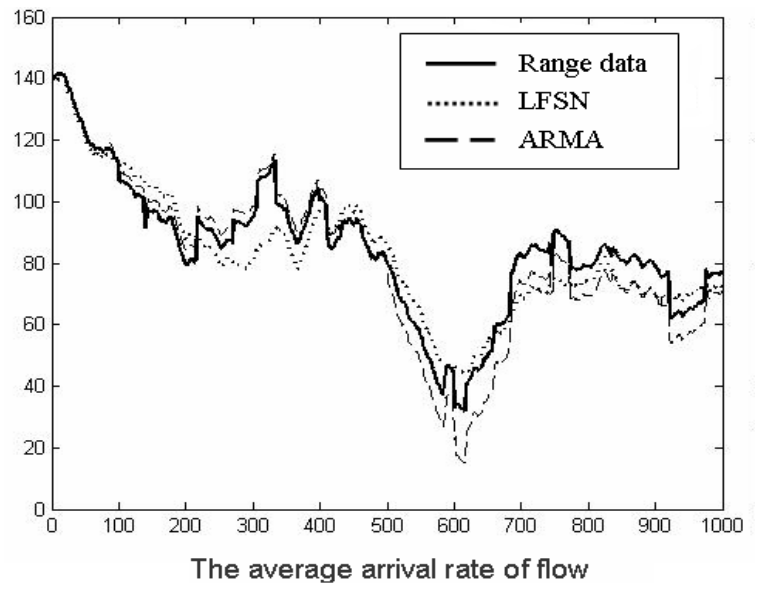

Fig. 2 Comparison of data and related two results that $\mathrm{H}=0.9$, figure 3 shows the average change rate between the effectiveness and the flow of a network node. From the overall situation, the effectiveness of node of a decreases with the increase of the average arrival rate. When the average arrival rate is small, the effectiveness of ARAMA corresponding to the larger; and when the average arrival rate is large, curve has a jump, the effectiveness of SNLF corresponding to the larger. This shows that in the arrival rate is small, using ARAMA model will get better performance, and the performance is better arrival rate under the SNLF model. 


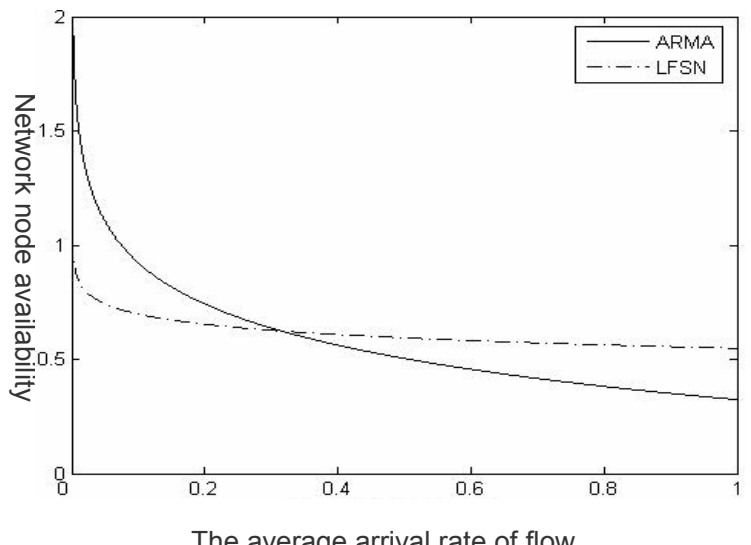

Fig. 3 Average arrival rate between

the network node availability and traffic

May see from the above research, there are more factors affecting the effectiveness with the Hurst parameter, node average velocity of flow, so should consider various factors to describe.

\section{Conclusion}

Aiming at the congestion caused by node failure in the network, combined with related characteristics of actual flow, put forward the evaluation index to measure the validity of the nodes, and through the ARAMA model and LNFS model respectively, the index is studied. Among them, the ARAMA method using the wavelet transform to reduce the negative influence brought by the long correlation, prediction method was established for the LNFS; and based on Fractal stable motion derived alpha stable boundary of the average length of the actual arrival flow. At the same time, the two methods are compared through simulation experiment, and in-depth study of the relationship between the factors of node effectively and its influence. In a follow-up study, considering the FARIMA model, the formation of network availability relatively perfect evaluation system.

\section{Reference}

[1] Sahinoglu M., Libby D. L.. Measuring availability indexes with small samples for component and network reliability using the Sahinoglu-Libby probability model[J]. IEEE Transactions on Instrumentation and Measurement, 2005, 54 (3): $1283-1295$.

[2] Ding Chang-song, Hu Zhi-gang, Xiao Peng. Reliability enhenced resource reservation strategy in computing grid[J]. Journal on Communications, 2011,32 (7): 40-46.[Ding song Chang, Hu Zhigang, Xiao Peng. The reliability enhancement of resources in grid environment strategy [J]. Journal of China Institute of communications, 2011,32 (7): 40-46.]

[3] Lin Y. K.. Reliability of a computer network in case capacity weight varying with arcs, nodes and types of commodity[J]. Reliability Engineering and System Safety, 2007, 92 (5): 646-652.

[4] Gunawan I.. Redundant paths and reliability bounds in gamma networks[J]. Applied Mathematical Modeling, 2008,32 (4): $588-594$.

[5] Xiaolong Wu, Shahram Latifi. Substar reliability analysis in star networks[J]. Information Sciences, 2008,178 (10): $2337-2348$.

[6] Felemban E., Lee C. G., Ekici E.. MMSPEED: multi-path multi-SPEED protocol for QoS guarantee of reliability and timeliness in wireless sensor networks[J]. IEEE Trans. Mobile Computing, 2006, 5 (6): 738-754.

[7] Tan Xianhai, Huang Yuanhui, Jin Weidong. Random Early Detection Algorithm of Self-sim ilar Traffic[J]. Journal of Southwest Jiaotong University, 2008, 43 (1): 19-24.[Xianhai Tan, Huang Yuanhui, Jin Weidong. The self-similar flow random early detection method [J]. Journal of Southwest Jiao Tong University, 2008, 43 (1): 19-24.]

[8] Lazarou G. Y., Baca Julie, Frostv S., et al. Describing network traffic using the index of variability[J]. IEEE /ACM Transactions on Networking, 2009, 17 (5): 1672-1683.

[9] Rao Yun-hua, Cao Yang, Yang Yan, Wang Xi-wen. Performance analysis of network queue based on FARIMA model. Computer Engineering, 2006, 32 (23): 13-20.[Yunhua Rao, Cao Yang, Yang Yan, Wang Xiwen. Model of FARIMA Network Queuing Performance Analysis Based on [J]. Computer Engineering, 2006, 32 (23): 13-20.]

[10] Miguel Lopez-Guerrero, Luis Orozco-Barbosa, Dimitrios Makrakis. Probabilistic envelope processes for self-similar traffic models and their -stable application to resource provisioning[J]. Performance Evaluation, 2005, 61 (2-3): 257-279.

[11] Gao Bo, Zhang Qin-yu, Liang Yong-sheng, et al. Predicting self-similar networking traffic based on EMD and ARAMA[J]. Journal on Communications, 2011, 32 (4): 47-56. [high waves, Zhang Qinyu, Liang Yongsheng, et al. EMD and ARAMA self-similar network traffic prediction based on [J]. Journal of communication, 2011, 32 (4): 47-56.]

[12] Sun Cheng-jie Liu Feng Lin Lei Liu Bing-quan. Prediction of Search Data Volume Based on Time-Series Clustering and ARAMA Models[J]. Journal Of South China University of Technology (Natural Science Edition), 2011, 39 (4): 21-25. [sun Cheng Jie, Liu Feng, Lin Lei, Liu Bingquan. Prediction of time series clustering and retrieval based on ARAMA model [J]. Journal of South China University of Technology (NATURAL SCIENCE EDITION), 2011, 39 (4): 21-25.]

[13] Vinay J.Ribeiro, Rudolf H. Riedi and Richard G.Baraniuk. Multiscale queueing analysis[J]. IEEE/ACM TRANSACTIONS ON NETWORKING, 2006, 14 (5): 1005-1018.

[14] [Xu Xiaodong, Zhu Shirui, Sun Yamin. Fractal characteristics of large-scale network traffic anomaly analysis based on [J]. Journal of communication, 2009, 30 (9): 43-53.]Xu Xiao-dong, Zhu Shi-rui, Sun Ya-min. Anomaly detection algorithm based on fractal characteristics of large-scale network traffic[J]. Journal on Communications, 2009, 30 (9): 43-53.

[15] Bai Xiang-Yu, Ye Xin-Ming, Jiang Hai. Network traffic predicting based on wavelet transform and autoregressive model[J]. Computer Science, 2007, 34 (7): 47-54. [white Xiangyu, Ye Xinming. Based on the wavelet transform and the network flow model [J]. computer science, 2007, 34 (7): 47-54.]

[16] Tan Xianhai, Huang Yuanhui, Jin Weidong. Modeling and performance analysis of self-similar traffic based on FBM[C]. 2007 IFIP International Conference on Network and Parallel Computing Workshops, Berlin, Springer, 2007: 543-548. 